\title{
Editorial
}

\section{Toward the Philosophy of CAM: Super-system and Epimedical Sciences}

\author{
Tomio Tada \\ Honorary Founder Emeritus, Professor Emeritus, University of Tokyo, Japan
}

People commonly think of complementary and alternative medicine (CAM) as something negative, using terms such as 'unorthodox', 'non-conventional' or 'non-Western'. Even in positive terms, it usually sounds like something secondary, complementing orthodox modern Western medicine.

\section{Positive Concept of CAM?}

Let us now ask if it is possible to present the idea of CAM in other positive ways. Since more and more people are attracted to CAM, there must be a new point of view from which CAM can be defined affirmatively. Certainly, the people who are turning to CAM are not merely escaping from something: they must be hoping to enter a new horizon of medicine rather than accepting secondary non-orthodox remedies. What is then the central, positive idea of CAM on this new horizon?

Let us first pose a simple question: what is the object of, or who is the subject of CAM? It is now well appreciated that Nature is structured hierarchically. For example, starting from ourselves, descending orders will be seen from the beginning level of the individual, to cells, molecules, genes, chemical substances, atoms and finally down to subatomic particles and fields. Above and surrounding us are higher society of human-beings and living creatures, the ecosystem, the planet Earth, galaxies and up to the Universe itself. Clearly, what CAM wishes to face is the human being, who is at the 'individual' level.

\section{Nature's Hierarchy and Emergence of 'Individual'}

The laws of Nature's lower levels restrict those governing the higher level of its hierarchy, and many of the phenomena at the higher level are explained and restricted by the rules governing the lower. This is seen easily in the history of our understanding of 'disease'. In pre-modern eras, cultures regarded human illness as a misfortune or spell that had befallen or been cast upon the individual who was the patient. Around the 18th century, modern Western medicine, firmly based on the exact anatomy or analysis of the human body, introduced the concept of disease as a malfunction of organs (organ pathology), the lower level of the individual. In the next century, the object of pathology was further reduced down to the level of cells, which turned pathology into 'cellular pathology', as declared by Virchow. We are now well aware that the path was pursued further and further down in the 20th century, from cells to molecules, and now to genes. The last century was the century of molecular medicine, which in pathology was molecular pathology. The modern scientific understanding of diseases has been accomplished by reducing its object from the level of the individual down to those of organs, cells, molecules and genes.

It has been the great and powerful methodology of modern biomedicine that has allowed us to understand the individual's illness by finding elemental factors at levels lower than the individual. We all know, thanks to this approach, that we have succeeded in explaining, in terms of molecules, many diseases from their etiology to pathogenesis. This is really a remarkable accomplishment.

But it may be an illusion if we think we can understand phenomena on the higher level of Nature's hierarchy by reducing them to the lower level. To return to this epistemological question later, it is certainly false to think that a patient's illness has been healed whenever we correct malfunctions of his/her organs or molecules. On the contrary, in principle, a patient's illness is not necessarily healed even if his organs/ molecules are mended. Reductionism, though powerful, especially in biomedical sciences or analysis of disease mechanisms, is defective in clinical medical practice or the art of healing. It can even become dangerous as it lures people into forgetting that the disease as a malady of the individual, not molecules, is the object (or subject) of medical practice.

This practical wisdom has an epistemological basis. It is now well recognized that not all phenomena taking place at the higher, or more complex, level of Nature can be explained by the principles governing the lower, or less complex, levels, as any time when a higher level emerges in Nature, new rules governing that level will emerge. We should thus be reminded that there are emergent rules at the level of the individual that cannot be reduced to the levels of organs, cells and molecules.

Even between the levels of cells and molecules, the functions of the former cannot be reduced to the functions of the 
latter. At least there should be new rules at the level of the cell that control and govern the interactions between its constituent molecules. Cells could only survive under such rules of cooperation controlling the functions of an immense number of different molecules. The same would apply to the relationship between the levels of cells and organs. Although an organ's function depends on the function of cells that constitute it, its function is not reducible to that of any constituent cells, e.g. thinking cannot be explained by the function of any one neuron in the brain. An organ's function is not an arithmetic sum of its constituent cells. It operates at least on the rules governing the interactions of each cell and those controlling its interactions with other organs and the individual's internal and external environments.

Likewise, an individual human being is not a collection of organs. He or she is certainly not free from the rules controlling his or her organs, but his/her individuality is not explained away in terms of organs. An individual is governed by the rules which integrate the whole constituent organs to operate cooperatively. Let us thus be reminded here that illness is something an individual suffers from. It is the suffering of an individual, not malfunctions of his/her organs/ cells/molecules. Disease as suffering (maladie in French and $K u$ in Japanese) cannot be understood without staying firmly on the level of the individual.

\section{A Fresh Look at the Patient as an Individual}

Let us imagine a patient with chronic fatigue syndrome, a very obscure state of mind-body recently identified as (probably) a distinct disease entity that may have an infectious and/or immunological basis. His fatigue is real and he often feels helpless because of this. However, there is still no definite measure to represent this mind-body condition, e.g. blood chemistry or imaging, in modern Western medicine. Without any objective measures he still remains with his suffering. Reductionist medicine has no way to understand or heal him, as it should be based on the evidence represented by some objective measures concerning his cellular or molecular functions. Thus, it cannot understand or heal the suffering individual.

The situation is the same for those diseases that can be diagnosed but not healed by modern medicine, such as advanced cancer and AIDS. The patient's condition on the levels of organs, cells and molecules is well represented numerically or in images. But her suffering cannot be represented in those measures. It is only expressed at the level of the individual.

I believe people turning to CAM are searching for a way to heal their suffering that conventional reductionist medicine tends to overlook because of its biased attention to the objective measures at the levels lower than individual: of organs, cells and molecules. What is hoped for from CAM is an attentive look at the individual as a whole.

I would like to propose that CAM should first define itself as a fresh look at the individual patient, different from the attitude of modern analytical medicine. A patient as an individual cannot be represented as collections of objective numerical measures at the sub-individual levels. What is hoped for from CAM is to understand and heal disease, not as an abnormality of objective measures but as the suffering of a patient as an individual in totality. The pre-modern medical systems of Asia, such as Chinese herbal medicine and Ayurvedic medicine, should be revisited from this perspective. Lacking sophisticated analytical power, these systems have nurtured intuitive diagnostic and healing methods based not on the sub-individual pathology but phenomenological symptomatology at the level of the individual.

I believe that it has been the general approach of CAM practitioners to understand disease by empirical intuitive symptomatology, not reducing it to the abnormalities at subindividual levels. If we dispel the superstitious elements from such an approach, it will teach us of their clinical wisdom, the attitude that stays firmly above the level of organs, cells and molecules. Even modern 'Western' doctors, if they are good enough, intuitively possess this holistic attitude towards the individual patient. We have to bring this intuition up to a theoretical methodology.

The basic standpoint of CAM is that new principles will emerge, as any new level of hierarchy in Nature emerges, discontinuously. We should recognize that there is a discontinuous leap from the lower to higher levels of complexity in Nature. For example, for the leap from the level of material to that of life, there emerged the rule of self-replication, which is based on, but not explained by, the rules governing the level of molecules. Evolution, of which the central mechanism is differential replication of various self-replicators, is the phenomenon intrinsic to this level, namely life. This is an emergent phenomenon irreducible to the non-living material world, since it takes place as complex self-replicators in immense numbers interact with the non-self-replicating material environment (lower level) and among themselves.

Similarly, I would like to assert that new rules have emerged as cells are organized up to the higher level, namely the individual. Only with this recognition does it become possible to understand that disease is a problem at the level of the individual, not usually reducible to the lower levels of its constituents: organs, cells and molecules.

\section{What has Emerged at the Level of Individual?}

Let us now consider what has emerged at the level of individual. One human body consists of 270 kinds of cells, totaling 60 trillion. Though it is now possible to culture many of our cells under controlled conditions, not even one of our cells can function if left alone. They can only survive and function normally in the tissues and organs with circulating blood, which constitute the unified environment of one individual. Thus, rules governing the interactions among those cells and organization of the functional units are required to construct the totality of one individual. Even one single human cell has developed from one single original cell, the fertilized ovum, 
through adaptive differentiation and proliferation. I would like to propose that the principles of self-generation, selfdiversification and self-organization are the new principles governing the process in which the level of the individual has emerged. Another related principle would be that of selfreferentiality, by which I mean diversified cells and organs interacting with each other in such a manner that they are coordinated to behave as if referring to the totality of self. I have to apologize for introducing here, in addition to the concept of 'individual', the concept of 'self', which may sound mystical to those hard-wired reductionists. However, this concept, in fact, is not mystical at all but even conventional in several fields of biomedicine, such as immunology. Immunology is nothing but deciphering the biological mechanisms of one individual to differentiate and defend one's self from others and the environment (non-self). Self-referentiality is the principle most obviously seen operating in this system. Innate and adaptive immunity, inflammation and hemetopoiesis are just a few phenomena governed by this principle. Although the immunological self is the bodily or genomic self, there is in addition the self more appropriately called by this name: the personal or conscious self. Scientists, including myself, hope that this level will be explained in terms of neuro-endocrinology in the future. But today fierce philosophical debate is raging over whether this explanation is possible or not, even in principle, never mind in practice. That debate aside, most scientists would at least agree that phenomena related to the conscious self are hard to tackle by the reductionist approach.

The reason for this is rather obvious. The traditional reductionist approach is restricted by the Newtonian mechanistic paradigm where its 'system' model is formed around the clockwise mechanical engineering system. In the clockwise mechanistic system, elemental components are put together only on the laws of Newtonian mechanics of rigid bodies. This mechanical engineering model of 'system' is clearly inadequate for the level of life. The ancient healers in India and China had insight that there should be such a higher 'system' in life, sometimes called 'spirit' in English terms, though they were at the same time immersed in non-analytical superstitions. We have to admit that there is an element of wisdom in the awe expressed towards the higher level of an extremely complex system, in various traditional medicines throughout the world.

The concept of 'system', which became popular in the second half of the last century, was loosely formed to denote a collection of elements, the total of which are composed to serve a useful purpose. Originally, it was a concept of mechanical engineering, and afterwards of information technology. At present, the system concept is more sophisticated in computer imagery than clocks. However, even the supercomputer system is far from the immunological system, not to mention the nervous system. What makes the living system different from those engineering systems? This is a hot subject not yet resolved by the theory of complexity, but at least one of its obvious properties is the self-organization of ele- ments that are generated by self-replication. Such a property is not endowed to any Newtonian mechanical systems. And even the computer, that is, Turing machine, can mimic such a property only superficially. Self-organization, self-diversification, self-generation and self-referentiality: these are properties of the living system that are beyond those of man-made machines. We should also be reminded that these properties only become apparent above the level of individual living organisms, especially those developed from the egg. Being cautious about neologism but in order to make this point clear, I would like to propose calling such a system with these properties a 'super-system'. By introducing such a concept, we can now say that the living individual is a super-system. Incidentally, I would like to suggest that these super-system properties are carried over to any systems above individuals: language, society, market, etc.

\section{Super-system and Epimedical Science}

We can then propose that the diseases alternative medicine should heal are disorders of this super-system. It cannot be reduced to the dysfunctions of its constituent elements. The experiences of human battles against the disorders of the super-system have been accumulated in the traditional intuitive medicines of the East and West. In that history, we should search for Jewels for the non-reductionist, alternative approach of biomedicine of the future.

Let me consider one simple aspect, namely of therapeutic technique, to exemplify the non-reductionist approach in medicine. Kampo (Chinese) and Western herbal therapies are based on the therapeutic effects of natural products. Many of these effects are gone when the herbs are analyzed into their constituent components. For herbal healers, as well as for super-system theorists, it is no wonder that crude and complex extracts of natural products possess therapeutic properties that are not traced down to any of their purified components. It is not necessary to resort to 'holistic' language here. The interactions and synergy of multiple components can give rise to emergent effects. This is the most elementary level of the super-system approach.

While the methodology of Western medicine had not advanced much beyond isolation, purification and analysis of components, the super-system approach is viewed as almost magical. I hope that modern biomedicine is now prepared to tackle the problem of complexity, learning from the wisdom of traditional medicines.

I think science is much more interesting than magic, so understanding various CAM remedies not in terms of miracle but of science will be much more interesting and rewarding than advocating them as magic bullets. Magic or miracles aside, even many of the physiological actions of common foods cannot be reduced to their constituent molecules. For example, as recently shown, the anti-prostate cancer effect of tomatoes cannot be reduced to lycopene, its major component, but is due to some still-unknown complex interaction of various components. Eating natural foods for decades should 
be different from taking supplementary pills for months or even years.

I would like to propose that the impact of CAM, including traditional medicine, on modern biomedicine is that it opens up this perspective of the super-system. It is not one alternative, or much less, a complementary medicine to the present reductionist biomedicine. I have thought that to put forward this position more clearly some new term other than CAM is necessary. 'Integrative Medicine' is one option to which I do not raise many objections, but the term cannot convey the concept that the object (or subject) of this new biomedicine is the super-system. As the terms 'super' or 'hyper' are too awful to be followed by medicine and also 'meta-medicine' sounds too naughty, I would like to suggest that we modestly call it 'epimedical science'. If this term is acceptable, the science which aims to study the complexity of the super-system and its healing can collectively be called 'epimedical science'. It is of course a new word coined by attaching the suffix epi-, namely 'above' or 'upon', to medicine. By this I wish to propose that the new biomedicine should include the best of modern analytical medicine, but stand above it to view the human individual as an irreducible super-system. Also implicated in the term epi- is that, in addition to modern Western medicine, the best of the present CAM modalities will be selectively included. They are, besides what I have discussed so far, acupuncture, shiatsu (Japanese massage), aromatherapy, Western massage, Qi-Gong, Rolfing, herbal medicines and other therapeutic modes which have been seen only as second rate or even as non-citizens of the modern medical world. Science of the super-system will elucidate what should be learned from them. As terms such as 'paramedical' and 'epigenetic' already have definite notations, I would wish the term epimedical science to be accepted widely in the future.

\section{Evidential Basis for Epimedical Science}

In trying to establish epimedical science as a discipline of science and healing art of the super-system, the crucial task is to construct its evidential basis. Superstition, religious faith and credulity, I am afraid, have been supporting much of CAM so far. It is now time to dispel these elements from CAM, as there are so many people, organizations and companies who wish to exploit unreasonable profits from CAM as it is. A scientific standard should be established to re-evaluate CAM according to an evidential basis, in order to integrate it into the medicine of the future or epimedical science.

This journal, Evidence-based Complementary and Alternative Medicine (eCAM), has been established in part to provide a forum for the discussion of this aspect, namely what kind of methodology is to be adopted to evaluate the evidential basis of CAM. Although we believe that some of the present methodology, especially the double-blind, placebo-controlled randomized clinical trial, represents scientifically sound objective ways to evaluate the effect of a single component drug on a single, well-defined manifestation of a disease, we are still not convinced that it should be the only way for evaluating the effects of complex agents, such as herbs, on complex conditions, such as fatigue. Neither do we believe that an epidemiological technique of comparable rigor has been established for evaluating the effects of, say, acupuncture. A simple commonsensical reason for this is that the effects of these complex agents are highly variable among individuals, even when placebo effects are excluded.

Philosophically, this problem of methodology is related to the super-system concept itself. We should assume, according to the hierarchical structure of the super-system, that evidence at a certain level of hierarchy should not be taken as relevant for the phenomena at a higher level. Thus, collecting evidence at lower levels should not, however much is accumulated, be simply regarded to support or reject the effects seen at higher levels. For example, it has been claimed that several CAM regimens, like Qi-Gong, can improve one's general health by influencing the immune system, based on the data collected for, say, number and activities of natural killer (NK) cells. But we should think that this kind of argument is insufficient to prove the effects of complex agents on the supersystem, as it itself is based on the evidence at lower levels. Effects of complex agents on the super-system should be examined at the level of the individual. Different measures and markers should be explored to represent this level, and advanced statistical methods need to be developed which can take actual individuality into account.

I hope I have succeeded so far in providing at least some argument to show that the present CAM can be taken as the starting point towards the epimedical science of the supersystem, departing from modern analytical biomedical science, and not as something secondary or supplemental to it. With this concept, we aim together to construct a medicine for the future based firmly on the irreducible property of the individual as super-system, integrating the best of both analytical (modern) and intuitive (traditional) biomedical science as essential elements of epimedical sciences. I also hope that the journal $e C A M$ will become an indispensable tool for those who wish to participate in this formidable but enjoyable task. 


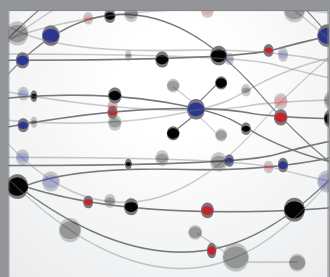

The Scientific World Journal
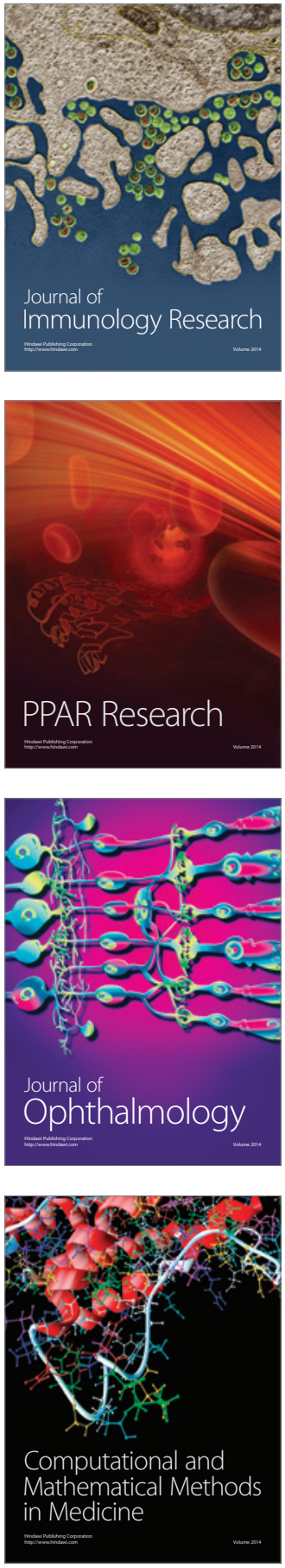

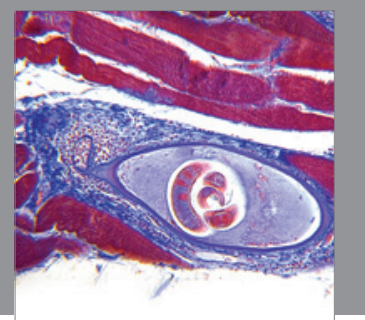

Gastroenterology

Research and Practice
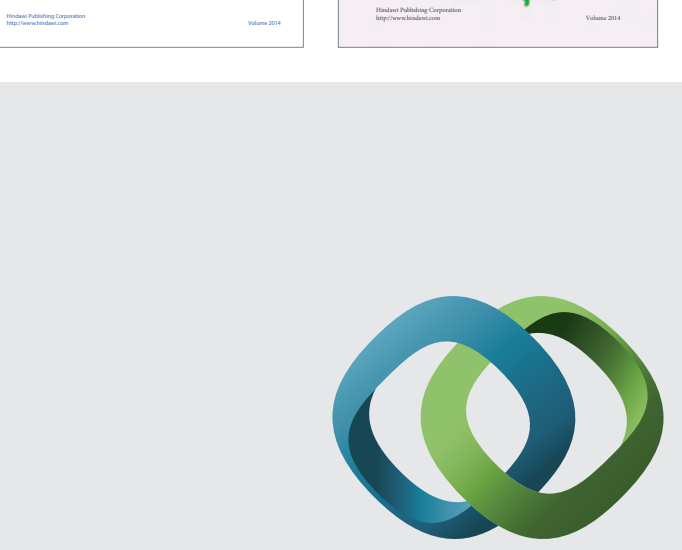

\section{Hindawi}

Submit your manuscripts at

http://www.hindawi.com
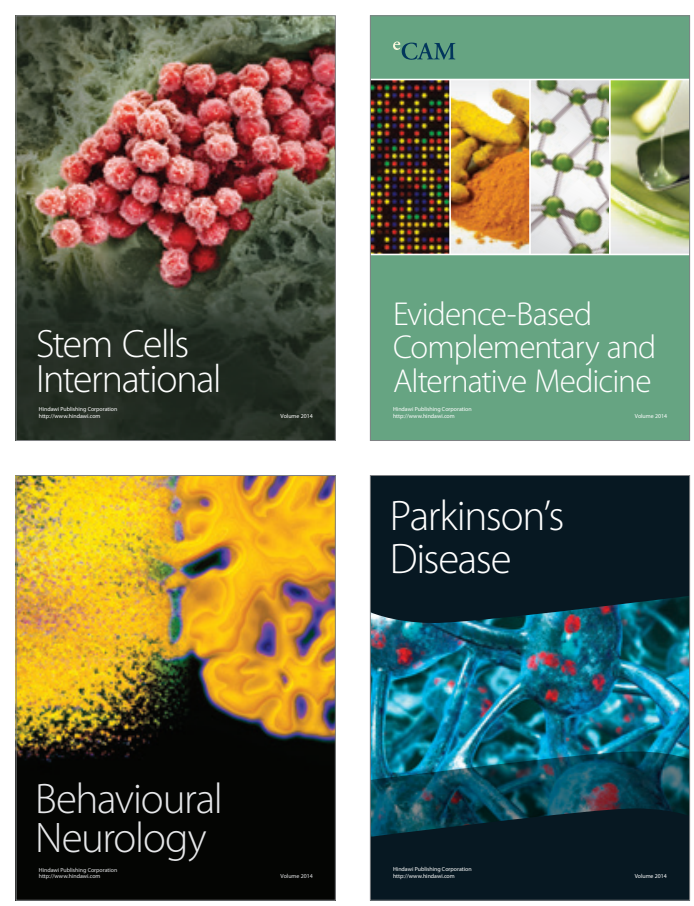

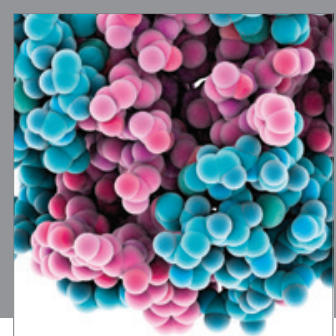

Journal of
Diabetes Research

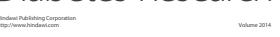

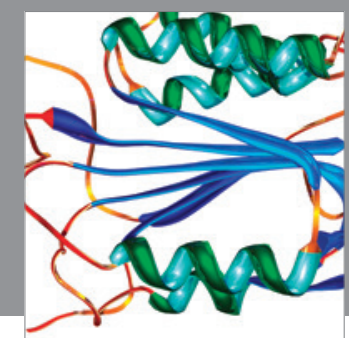

Disease Markers
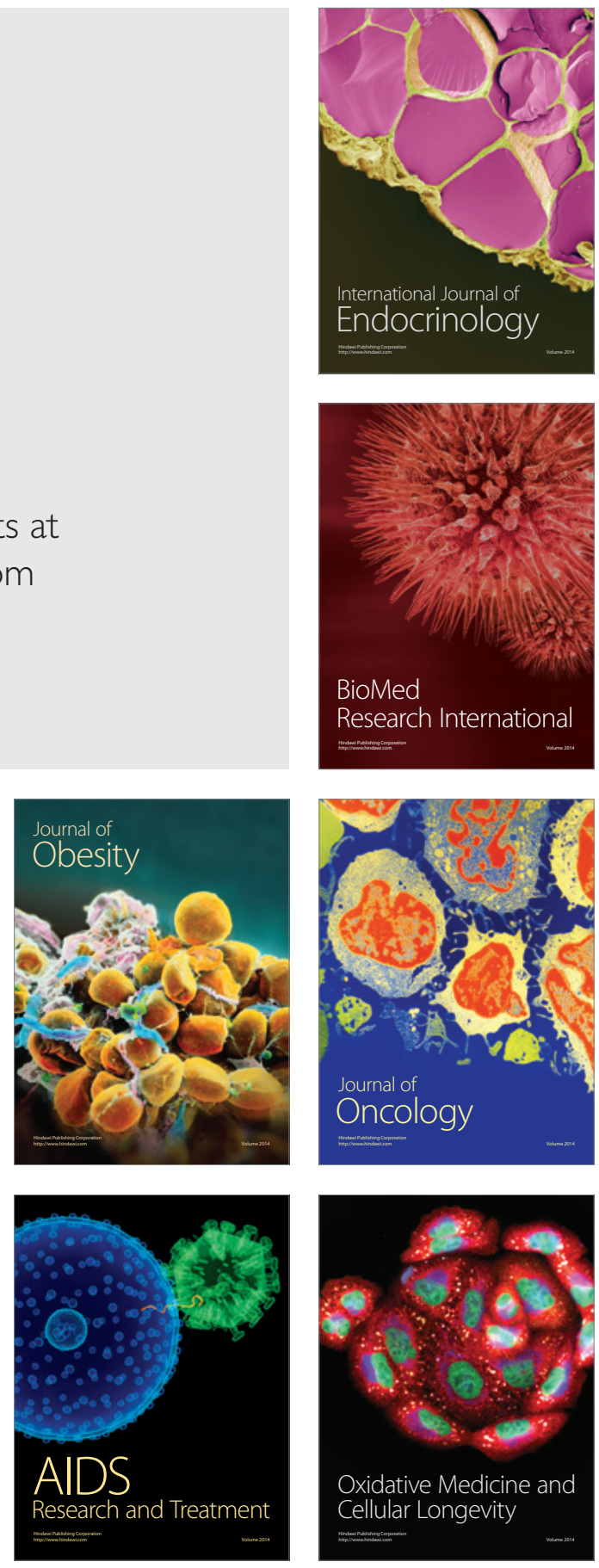